\title{
On attenuation of the seismic Rayleigh waves propagating in an elastic crustal layer over viscoelastic mantle
}

\author{
M NeGin ${ }^{1, *}$ (๑) and S D AKBAROV ${ }^{2,3}$ \\ ${ }^{1}$ Department of Civil Engineering, Bahcesehir University, Istanbul, Turkey. \\ ${ }^{2}$ Department of Mechanical Engineering, Yildiz Technical University, Istanbul, Turkey. \\ ${ }^{3}$ Institute of Mathematics and Mechanics of the National Academy of Sciences of Azerbaijan, Baku, Azerbaijan. \\ *Corresponding author.e-mail: masoud.negin@eng.bau.edu.tr
}

MS received 2 February 2018; revised 27 March 2019; accepted 14 April 2019; published online 29 June 2019

This study investigates the attenuation of the seismic Rayleigh waves propagating in an elastic crustal layer of the Earth over its viscoelastic mantle. The exact equations of motion of the theory of linear viscoelasticity are used and the complex dispersion equation is obtained for an arbitrary type of hereditary operator of the viscoelastic materials. The viscoelasticity of the materials is described by the fractionalexponential operators of Rabotnov, and a solution algorithm is developed to obtain numerical results for the attenuation of the considered waves. Attenuation curves are obtained and discussed, and in particular, the influence of the rheological parameters of the materials on this attenuation is studied. It is established that a decrease in the creep time of the viscoelastic materials leads to an increase in the attenuation coefficient.

Keywords. Seismic Rayleigh waves; wave attenuation; viscoelastic material; rheological parameters; fractional-exponential operator.

\section{Introduction}

It is known that the materials of the deep layers of the Earth are viscoelastic, causing seismic waves to dissipate as they propagate through the material. Consequently, any mathematical model used to simulate and study seismic waves must be able to accurately explain the effects of attenuation and dispersion of the propagated waves. The study of wave propagation in attenuative materials has been the subject of extensive investigation in the literature, and most of the related investigations before the 1990s can be found in the paper by Carcione (1992). More comprehensive theoretical and technical information is also given in monographs by Aki and Richards (2002) and Carcione
(2007). However, most of the research studies were conducted using simple mathematical models in the following two cases. In the first case, the viscoelasticity of the material has been described by spring-dashpot models such as the classical KelvinVoigt or Maxwell models, or some combination of these such as the SLS standard linear solid model (see, for instance, Eldred et al. 1995; Kielczyriski and Cheeke 1997; Simonetti and Cawley 2003; Carcione et al. 2004; Ely et al. 2008; Jiangong 2011; Manconi and Sorokin 2013; Chiriţă et al. 2014; Ivanov and Savova 2014; Quintanilla et al. 2015). In the second case, the real elasticity constants have been replaced with the complex modulus in the stress-strain relations of the viscoelastic materials (see, for instance, Carcione 1995; Castaings and 
Hosten 2003; Barshinger and Rose 2004; Addy and Chakraborty 2005; Garg 2007; Vishwakarma and Gupta 2012). Unfortunately, such simple mathematical models cannot illustrate the real and complex nature of the viscoelastic materials or the effect of the rheological parameters of the viscoelastic material on the dispersion and attenuation of the corresponding waves. Recent efforts by Meral et al. $(2009,2011)$ utilising the fractional order Voigt model are more realistic for wave propagation and attenuation problems in viscoelastic media. In this way, by introducing a new rheological parameter as the order of the fractional derivatives, they obtained analytical results that are in very good agreement with experiments compared to conventional models.

Here is a brief review of some studies on the dispersion and attenuation of Rayleigh waves in viscoelastic media, which are close to the subject of our study. Carcione (1992) investigated the anelastic characteristics of Rayleigh waves from the standpoint of energy balance. He calculated the quality factors as a function of the frequency and depth, and showed that the viscoelastic properties calculated from an energy consideration are consistent with those obtained from the Rayleigh secular equation. Romeo (2001) showed that the secular equation of Rayleigh waves propagating over the viscoelastic half-space always admits only one complex root corresponding to a surface wave. He obtained the roots in terms of complex integrals and showed that the wave solution represents an admissible surface wave for any viscoelastic relaxation kernel compatible with thermodynamics. Lai and Rix (2002), based on the Cauchy residue theorem of complex analysis, presented a technique that allows for the simultaneous determination of the Rayleigh dispersion and attenuation curves for linear viscoelastic media with arbitrary values of the material damping ratio. Jousset et al. (2004) studied the magma properties and rheology and their effects on low-frequency volcanic earthquakes. Using linear viscoelastic theory, they showed that volcanic media for seismic frequencies above $2 \mathrm{~Hz}$ can be approximated by a standard linear solid state (SLS). The results show that the attenuation changes both the amplitudes and the dispersive properties of low-frequency seismic waves, thus, the assumption of a SLS for low-frequency earthquakes fails. Fan (2004) examined the nonlinear damping mechanism of seismic waves by applying the perturbation method and obtained the analytical solution of Rayleigh wave propagation.
Pasternak (2008), using Biot's viscoelastic solid model and formulating the problem in FourierLaplace space, studied the Rayleigh wave propagation problem in an interface of the elastic half-space and viscoelastic layer. Zhou (2009) derived finitefrequency surface-wave sensitivity kernels of both phase delays and amplitudes to perturbations in an elasticity and showed that in the upper mantle this factor affects not only the amplitude of surface waves but also significantly affects the phase delay of surface waves. Zhang et al. (2011) investigated the dispersion of Rayleigh waves in viscoelastic media by applying the pseudospectral modelling method to obtain high accuracy. In the pseudospectral method, spatial derivatives in the vertical and horizontal directions are calculated using Chebyshev and Fourier difference operators, respectively. Chiriţă et al. (2014) studied the propagation of surface waves over an exponentially graded halfspace of the Kelvin-Voigt isotropic viscoelastic material using wave solutions with spatial and temporal finite energy. They showed that when there is only one wave solution, it is retrograde at the free surface, whereas when there is more than one viscoelastic surface wave, one is retrograde and the others are direct to the free surface. Quintanilla et al. (2015) studied the dispersion of guided waves propagating in anisotropic single- and multilayered viscoelastic media in a flat and cylindrical geometry. For material damping, they used KelvinVoigt and hysteretic models in various cases and validated their results in comparison with those from semi-analytical finite element simulations. Chen et al. (2015) studied the Rayleigh-type surface waves and their properties in two-dimensional viscoelastic media by applying the finite-difference method in a time-space domain. They compared the analytical and numerical solutions and showed that, in a shallow subsurface, the phase velocity of a fundamental mode of Rayleigh waves increases considerably as the quality factor $Q$ decreases. Yuan et al. (2018) also investigated the attenuation and dispersion characteristics of Rayleigh waves in viscoelastic media through finite-difference modelling. They compared elastic and viscoelastic modelling results in terms of wave-field snapshots, synthetic seismograms and dispersive images.

From the foregoing discussion and studies reviewed thereafter, it is clear that the research on the dispersion and attenuation of guided waves in elastic-viscoelastic-layered half-spaces, for the most part, has been carried out either by replacing the real elasticity modulus of the materials 
with the frequency-independent (the hysteretic model) or frequency-dependent complex modulus (the Maxwell, Kelvin-Voigt or SLS models) obtained from the experiments. Consequently, the influence of the rheological parameters of the viscoelastic materials on the dispersion and attenuation of the propagated waves has not been considered in these simple models. Therefore, they cannot actually describe the real and complicated behaviour of viscoelastic materials. These considerations directed the authors to study the near-surface wave dispersion and attenuation of a viscoelastic covered half-space utilising a more realistic mathematical model (Akbarov and Negin 2017b) by using Rabotnov (1980) fractionalexponential operators which are used in the papers by Akbarov (2014, 2015), Akbarov and Kepceler (2015), Akbarov et al. (2016a,b) and Kocal and Akbarov (2017) in the first place. This study, in that sense, is actually an extension of the authors' previous studies (Negin et al. 2014; Negin 2015; Akbarov and Negin 2017a) on dispersion of the generalised Rayleigh waves in an initially stressed elastic covered half-space to the viscoelastic case, where the constitutive relations of the materials of the covering layer and the half-space are described through the fractional-exponential operator by Rabotnov (1980). A review of the fractional-exponential operator, proposed for the first time by Rabotnov, is given in the monograph by Rabotnov (1980) and in the papers by Rossikhin (2010), Bosiakov (2014), Rossikhin and Shitikova (2014) and others listed therein.

Though the dispersion of Rayleigh waves in a viscoelastic-covered half-space was studied in the paper by Akbarov and Negin (2017b), for the selected wave attenuation cases, we have not found any investigation carried out so far utilising the fractional-exponential operators by Rabotnov (1980) or studying the dispersive attenuation of viscoelastic Rayleigh waves for the selected possible dispersion curves. Thus, the main objectives of the present study can be formulated as follows:

(i) formulation of the problem and development of the analytical-numerical approach for the theoretical investigation of the dispersive attenuation of the seismic generalised Rayleigh waves propagating in a system consisting of purely elastic covering layer and viscoelastic halfspace (which can be taken as a model for an elastic crustal layer of the Earth over its viscoelastic mantle); (ii) determination of the influence of the rheological parameters of the viscoelastic materials of the half-space on the attenuation of the aforementioned Rayleigh waves.

The investigations are carried out within the framework of the piecewise homogeneous body model. Exact equations of the linear theory of viscoelasticity are used and it is assumed that perfect interface conditions take place between the covering layer and the half-space. Numerical results and discussions on the influence of the rheological parameters of the viscoelastic materials on the attenuation of the seismic Rayleigh waves propagating in an elastic crustal layer of the Earth over the viscoelastic mantle are established. The theoretical results obtained in this study can be used in the geophysical sciences and seismic studies as well as in many practical engineering problems related to wave propagation in elastic-viscoelastic-layered media.

\section{Field equations and relations}

The geometry of the covering half-space is shown in figure 1. We assume that the thickness of the covering layer is $h$. The positions of the points are determined with the coordinates in the Cartesian system $O x_{1} x_{2} x_{3}$ associated with the interface plane between the covering layer and half-plane. We assume that the plane-strain state in the $O x_{1} x_{2}$ plane occurs in the considered covering layer + half-plane system, thus, the displacement components along the $O x_{1}$ and $O x_{2}$ directions, namely $u_{1}$ and $u_{2}$, are non-zero while the component of the displacement vector in the $O x_{3}$ axis direction is equal to zero. Moreover, we assume that the materials of the constituents of the system are isotropic, homogeneous and hereditary-viscoelastic (i.e., the response of a viscoelastic medium at any point

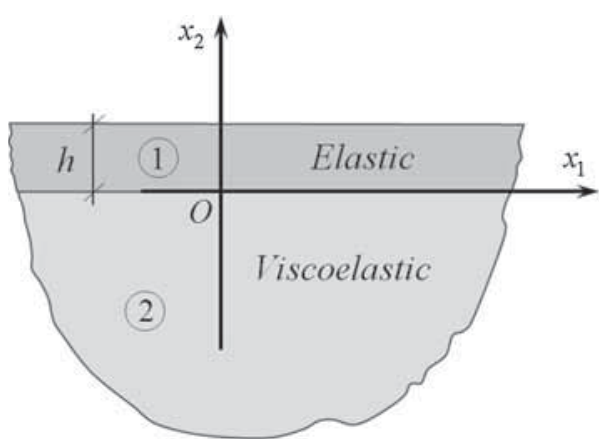

Figure 1. Geometry of the covered half-space. 
depends not only on the value of the action at that instant, but also on the complete history of all past actions). The near-surface waves propagate in the positive direction of the $O x_{1}$ axis. The governing field equations and relations can be written as follows:

- Equations of motion:

$$
\begin{aligned}
& \frac{\partial \sigma_{11}^{(m)}}{\partial x_{1}}+\frac{\partial \sigma_{12}^{(m)}}{\partial x_{2}}=\rho^{(m)} \frac{\partial^{2} u_{1}^{(m)}}{\partial t^{2}}, \\
& \frac{\partial \sigma_{12}^{(m)}}{\partial x_{1}}+\frac{\partial \sigma_{22}^{(m)}}{\partial x_{2}}=\rho^{(m)} \frac{\partial^{2} u_{2}^{(m)}}{\partial t^{2}}, \quad m=1,2 .
\end{aligned}
$$

- Constitutive relations and strain-displacement relations:

$$
\begin{aligned}
\sigma_{11}^{(m)} & =\lambda^{(m)^{*}} \theta^{(m)}+2 \mu^{(m)^{*}} \varepsilon_{11}^{(m)}, \\
\sigma_{22}^{(m)} & =\lambda^{(m)^{*}} \theta^{(m)}+2 \mu^{(m)^{*}} \varepsilon_{22}^{(m)}, \\
\sigma_{12}^{(m)} & =2 \mu^{(m)^{*}} \varepsilon_{12}^{(m)}, \\
\varepsilon_{11}^{(m)} & =\frac{\partial u_{1}^{(m)}}{\partial x_{1}}, \\
\varepsilon_{22}^{(m)} & =\frac{\partial u_{2}^{(m)}}{\partial x_{2}}, \\
\varepsilon_{12}^{(m)} & =\frac{1}{2}\left(\frac{\partial u_{1}^{(m)}}{\partial x_{2}}+\frac{\partial u_{2}^{(m)}}{\partial x_{1}}\right), \\
\theta^{(m)} & =\varepsilon_{11}^{(m)}+\varepsilon_{22}^{(m)},
\end{aligned}
$$

where $\lambda^{(m)^{*}}$ and $\mu^{(m)^{*}}$ are the following viscoelastic operators:

$$
\begin{aligned}
\left\{\begin{array}{l}
\lambda^{(m)^{*}} \\
\mu^{(m)^{*}}
\end{array}\right\} \varphi(t)= & \left\{\begin{array}{l}
\lambda_{0}^{(m)} \\
\mu_{0}^{(m)}
\end{array}\right\} \varphi(t) \\
& +\int_{0}^{t}\left\{\begin{array}{c}
\lambda_{1}^{(m)}(t-\tau) \\
\mu_{1}^{(m)}(t-\tau)
\end{array}\right\} \varphi(\tau) \mathrm{d} \tau .
\end{aligned}
$$

Here $\varphi(t)$ is an arbitrary function and $\lambda_{0}^{(m)}$ and $\mu_{0}^{(m)}$ are the instantaneous values of Lame's constants at $t=0$ and $\lambda_{1}^{(m)}(t)$ and $\mu_{1}^{(m)}(t)$ are the corresponding kernel functions describing the hereditary properties of the $m$ th material of the constituents (the explicit forms of equation 3 will be given in section 4 , equation 17 ). The other notation used in equations (1-3) is conventional.

According to figure 1, we assume that the following boundary and contact conditions on the free face plane of the covering layer and on the interface between the covering layer and half-space are satisfied:

- Boundary conditions:

$$
\left.\sigma_{12}^{(1)}\right|_{x_{2}=h}=0,\left.\quad \sigma_{22}^{(1)}\right|_{x_{2}=h}=0
$$

- Contact conditions:

$$
\begin{array}{ll}
\left.u_{1}^{(1)}\right|_{x_{2}=0}=\left.u_{1}^{(2)}\right|_{x_{2}=0}, & \left.u_{2}^{(1)}\right|_{x_{2}=0}=\left.u_{2}^{(2)}\right|_{x_{2}=0}, \\
\left.\sigma_{12}^{(1)}\right|_{x_{2}=0}=\left.\sigma_{12}^{(2)}\right|_{x_{2}=0}, & \left.\sigma_{22}^{(1)}\right|_{x_{2}=0}=\left.\sigma_{22}^{(2)}\right|_{x_{2}=0} .
\end{array}
$$

- Moreover, the following decay conditions must be satisfied:

$$
\left.\sigma_{i j}^{(2)}\right|_{x_{2} \rightarrow-\infty} \rightarrow 0,\left.\quad u_{i}^{(2)}\right|_{x_{2} \rightarrow-\infty} \rightarrow 0
$$

This completes the discussion of the field equations and relations that govern the present study.

\section{Solution of the field equations and obtaining the dispersion equation}

As we consider the harmonic waves propagating in the $O x_{1}$ direction, the components of the displacement vector and strain tensor can be presented as follows:

$$
\begin{aligned}
u_{i}^{(m)} & =v_{i}^{(m)}\left(x_{2}\right) \mathrm{e}^{\mathrm{i}\left(k x_{1}-\omega t\right)}, \\
\varepsilon_{i j}^{(m)} & =\gamma_{i j}^{(m)}\left(x_{2}\right) \mathrm{e}^{\mathrm{i}\left(k x_{1}-\omega t\right)}, \\
\gamma_{i j}^{(m)} & =\frac{1}{2}\left(\frac{\partial v_{i}^{(m)}}{\partial x_{j}}+\frac{\partial v_{j}^{(m)}}{\partial x_{i}}\right), \quad i, j=1,2 .
\end{aligned}
$$

After some mathematical operations, we can perform the following manipulations for the integrals in equation (3),

$$
\begin{aligned}
& \int_{-\infty}^{t} \lambda_{1}^{(m)}(t-\tau) \mathrm{e}^{-\mathrm{i} \omega \tau} \mathrm{d} \tau=\mathrm{e}^{-\mathrm{i} \omega t}\left(\lambda_{1 c}^{(m)}+\mathrm{i} \lambda_{1 s}^{(m)}\right), \\
& \int_{-\infty}^{t} \mu_{1}^{(m)}(t-\tau) \mathrm{e}^{-\mathrm{i} \omega \tau} \mathrm{d} \tau=\mathrm{e}^{-\mathrm{i} \omega t}\left(\mu_{1 c}^{(m)}+\mathrm{i} \mu_{1 s}^{(m)}\right),
\end{aligned}
$$


where

$$
\begin{aligned}
& \lambda_{1 c}^{(m)}=\int_{0}^{\infty} \lambda_{1}^{(m)}(s) \cos (\omega s) \mathrm{d} s, \\
& \lambda_{1 s}^{(m)}=\int_{0}^{\infty} \lambda_{1}^{(m)}(s) \sin (\omega s) \mathrm{d} s, \\
& \mu_{1 c}^{(m)}=\int_{0}^{\infty} \mu_{1}^{(m)}(s) \cos (\omega s) \mathrm{d} s, \\
& \mu_{1 s}^{(m)}=\int_{0}^{\infty} \mu_{1}^{(m)}(s) \sin (\omega s) \mathrm{d} s .
\end{aligned}
$$

Taking these relations into consideration, we finally obtain the following expressions for the stresses from equations (2 and 3 ):

$$
\begin{aligned}
& \sigma_{11}^{(m)}=\left[\Lambda^{(m)}(\omega) \vartheta^{(m)}\left(x_{2}\right)\right. \\
& \left.+2 M^{(m)}(\omega) \gamma_{11}^{(m)}\left(x_{2}\right)\right] \mathrm{e}^{\mathrm{i}\left(k x_{1}-\omega t\right)}, \\
& \sigma_{22}^{(m)}=\left[\Lambda^{(m)}(\omega) \vartheta^{(m)}\left(x_{2}\right)\right. \\
& \left.+2 M^{(m)}(\omega) \gamma_{22}^{(m)}\left(x_{2}\right)\right] \mathrm{e}^{\mathrm{i}\left(k x_{1}-\omega t\right)}, \\
& \sigma_{12}^{(m)}=2 M^{(m)}(\omega) \gamma_{12}^{(m)}\left(x_{2}\right) \mathrm{e}^{\mathrm{i}\left(k x_{1}-\omega t\right)},
\end{aligned}
$$

where

$$
\begin{gathered}
\Lambda^{(m)}(\omega)=\lambda_{0}^{(m)}+\lambda_{1 c}^{(m)}(\omega)+\mathrm{i} \lambda_{1 s}^{(m)}(\omega), \\
M^{(m)}(\omega)=\mu_{0}^{(m)}+\mu_{1 c}^{(m)}(\omega)+\mathrm{i} \mu_{1 s}^{(m)}(\omega) .
\end{gathered}
$$

In this way, instead of the real Lame constants in relations (2) and (3) we obtain the complex modulus $\Lambda^{(m)}(\omega)$ and $M^{(m)}(\omega)$, where their real and imaginary parts are determined through expressions (9) and (11). In other words, for the case considered, the complete system of field equations for the viscoelastic medium can also be obtained from the corresponding equations for the purely elastic system by simply replacing the elastic Lame constants $\lambda_{0}^{(m)}$ and $\mu_{0}^{(m)}$ with the complex modulus $\Lambda^{(m)}(\omega)$ and $M^{(m)}(\omega)$, respectively.

Now according to equations $(1,7,10$ and 11$)$, we obtain the following equations of motion in terms of the displacement amplitudes:

$$
\begin{aligned}
& M^{(m)} \frac{\mathrm{d}^{2} v_{1}^{(m)}}{\mathrm{d} x_{2}^{2}}+\left(\Lambda^{(m)}+M^{(m)}\right) \mathrm{i} k \frac{\mathrm{d} v_{2}^{(m)}}{\mathrm{d} x_{2}} \\
& \quad+\left(-\Lambda^{(m)}-2 M^{(m)}+\frac{\omega^{2}}{k^{2}} \rho^{(m)}\right) k^{2} v_{1}^{(m)}=0, \\
& \left(\Lambda^{(m)}+2 M^{(m)}\right) \frac{\mathrm{d}^{2} v_{2}^{(m)}}{\mathrm{d} x_{2}^{2}}
\end{aligned}
$$

$$
\begin{aligned}
& +\left(\Lambda^{(m)}+M^{(m)}\right) \mathrm{i} k \frac{\mathrm{d} v_{1}^{(m)}}{\mathrm{d} x_{2}} \\
& +\left(-M^{(m)}+\frac{\omega^{2}}{k^{2}} \rho^{(m)}\right) k^{2} v_{2}^{(m)}=0 .
\end{aligned}
$$

After some mathematical operations, we derive the following equation for $v_{2}^{(m)}$ :

$$
\begin{aligned}
\frac{\mathrm{d}^{4} v_{2}^{(m)}}{\mathrm{d}\left(k x_{2}\right)^{4}}+B_{2}^{(m)} \frac{\mathrm{d}^{2} v_{2}^{(m)}}{\mathrm{d}\left(k x_{2}\right)^{2}}+C_{2}^{(m)} v_{2}^{(m)}=0, & c_{2}^{(m)}=b_{22}^{(m)}+b_{21}^{(m)}-c_{21}^{(m)} c_{22}^{(m)}, \\
C_{2}^{(m)} & =b_{21}^{(m)} b_{22}^{(m)}, \\
b_{21}^{(m)} & =\frac{-\Lambda^{(m)}-2 M^{(m)}+\frac{\omega^{2}}{k^{2}} \rho^{(m)}}{M^{(m)}}, \\
c_{21}^{(m)} & =\frac{\left(\Lambda^{(m)}+M^{(m)}\right) \mathrm{i}}{M^{(m)}}, \\
b_{22}^{(m)} & =\frac{-M^{(m)}+\frac{\omega^{2}}{k^{2}} \rho^{(m)}}{\Lambda^{(m)}+2 M^{(m)}}, \\
c_{22}^{(m)} & =\frac{\left(\Lambda^{(m)}+M^{(m)}\right) \mathrm{i}}{\Lambda^{(m)}+2 M^{(m)}} .
\end{aligned}
$$

The general solution of equation (13) can be written as:

$$
\begin{aligned}
v_{2}^{(1)}\left(x_{2}\right)= & Z_{1}^{(1)} \exp \left(R_{1}^{(1)} k x_{2}\right) \\
& +Z_{2}^{(1)} \exp \left(-R_{1}^{(1)} k x_{2}\right) \\
& +Z_{3}^{(1)} \exp \left(R_{2}^{(1)} k x_{2}\right) \\
& +Z_{4}^{(1)} \exp \left(-R_{2}^{(1)} k x_{2}\right), \\
v_{2}^{(2)}\left(x_{2}\right)= & Z_{1}^{(2)} \exp \left(R_{1}^{(2)} k x_{2}\right) \\
& +Z_{3}^{(2)} \exp \left(R_{2}^{(2)} k x_{2}\right),
\end{aligned}
$$

where

$$
\begin{aligned}
& R_{1}^{(m)}=\sqrt{-\frac{B_{2}^{(m)}}{2}+\sqrt{\frac{\left(B_{2}^{(m)}\right)^{2}}{4}-C_{2}^{(m)}}}, \\
& R_{2}^{(m)}=\sqrt{-\frac{B_{2}^{(m)}}{2}-\sqrt{\frac{\left(B_{2}^{(m)}\right)^{2}}{4}-C_{2}^{(m)}} .}
\end{aligned}
$$

In a similar way, we can also determine the function $v_{1}^{(m)}\left(x_{2}\right)$ from equation (12). Note that as we consider the surface waves, according to the decay conditions in equation $(6)$, both $\operatorname{Re}\left(R_{1}^{(2)} k\right)<$ 
0 and $\operatorname{Re}\left(R_{2}^{(2)} k\right)<0$ inequalities must be satisfied simultaneously.

Finally, using expressions (14, 7 and 2) we obtain the following dispersion equation from the boundary and contact conditions (4 and 5):

$$
\operatorname{det}\left\|\alpha_{i j}\right\|=0, \quad i, j=1,2, \ldots, 6 .
$$

This completes the consideration of the solution to the field equations and obtaining the dispersion equation (16).

\section{Numerical results and discussions}

\subsection{Viscoelastic operators and dimensionless rheological parameters}

We recall that operators (3) describe the viscoelastic properties of the $m$ th material. However, to solve the dispersion equation, we need the explicit values of $\lambda_{1 c}^{(m)}, \lambda_{1 s}^{(m)}, \mu_{1 c}^{(m)}$ and $\mu_{1 s}^{(m)}$ by the kernel functions $\lambda_{1}^{(m)}$ and $\mu_{1}^{(m)}$ of the viscoelastic operators. Consequently, it is necessary to have explicit expressions for the kernel functions $\lambda_{1}^{(m)}(t)$ and $\mu_{1}^{(m)}(t)$ to determine the values of $\lambda_{1 c}^{(m)}, \lambda_{1 s}^{(m)}, \mu_{1 c}^{(m)}$ and $\mu_{1 s}^{(m)}$. As mentioned above, we describe the viscoelasticity of the materials through the fractionalexponential operator by Rabotnov (1980), i.e., we assume that

$$
\begin{aligned}
\lambda^{(m) *} \varphi(t)= & \lambda_{0}^{(m)}\left[\varphi(t)-\frac{\beta_{0}^{(m)}}{\left(1+\nu_{0}^{(m)}\right)}\right. \\
& \left.R_{\alpha^{(m)}}^{(m) *}\left(-\frac{3 \beta_{0}^{(m)}}{2\left(1+\nu_{0}^{(m)}\right)}-\beta_{\infty}^{(m)}\right) \varphi(t)\right], \\
\mu^{(m) *} \varphi(t)= & \mu_{0}^{(m)}\left[\varphi(t)-\frac{3 \beta_{0}^{(m)}}{2\left(1+\nu_{0}^{(m)}\right)}\right. \\
& \left.R_{\alpha^{(m)}}^{(m) *}\left(-\frac{3 \beta_{0}^{(m)}}{2\left(1+\nu_{0}^{(m)}\right)}-\beta_{\infty}^{(m)}\right) \varphi(t)\right],
\end{aligned}
$$

where

$$
\begin{aligned}
& R_{\alpha^{(m)}}^{(m) *}\left(x^{(m)}\right) \varphi(t) \\
& =\int_{0}^{t} R_{\alpha^{(m)}}^{(m)}\left(x^{(m)}, t-\tau\right) \varphi(\tau) \mathrm{d} \tau, \\
& R_{\alpha^{(m)}}^{(m)}\left(x^{(m)}, t\right) \varphi(t) \\
& =t^{-\alpha^{(m)}} \sum_{n=0}^{\infty} \frac{\left(x^{(m)}\right)^{n} t^{n\left(1-\alpha^{(m)}\right)}}{\Gamma\left((1+n)\left(1-\alpha^{(m)}\right)\right)} .
\end{aligned}
$$

Here, $\Gamma(x)$ is the Gamma function and the constants $\alpha^{(m)}, \beta_{0}^{(m)}$ and $\beta_{\infty}^{(m)}$ are the rheological parameters of the viscoelastic material.
The mechanical meanings of these rheological parameters are explained in the papers by Akbarov (2014) and Akbarov and Kepceler (2015).

Finally, using relations (9 and 18), we obtain:

$$
\begin{aligned}
\lambda_{c}^{(m)}= & \lambda_{0}^{(m)}\left[1-\frac{1}{\left(1+\nu_{0}^{(m)}\right)}\left(d^{(m)}+\frac{3 \beta_{0}^{(m)}}{2\left(1+\nu_{0}^{(m)}\right)}\right)^{-1}\right. \\
& \left.R_{\left.\alpha^{(m)}\right)_{c}}^{(m)}\left(-\beta_{01}^{(m)}-\beta_{\infty}^{(m)}, \omega\right)\right] \\
\lambda_{s}^{(m)}= & \lambda_{0}^{(m)} \frac{1}{\left(1+\nu_{0}^{(m)}\right)}\left(d^{(m)}+\frac{3 \beta_{0}^{(m)}}{2\left(1+\nu_{0}^{(m)}\right)}\right)^{-1} \\
& R_{\alpha^{(m)}}^{(m)}\left(-\beta_{01}^{(m)}-\beta_{\infty}^{(m)}, \omega\right), \\
\mu_{c}^{(m)}= & \mu_{0}^{(m)}\left[1-\frac{3}{2\left(1+\nu_{0}^{(m)}\right)}\left(d^{(m)}+\frac{3 \beta_{0}^{(m)}}{2\left(1+\nu_{0}^{(m)}\right)}\right)^{-1}\right. \\
& R_{\left.\left.\alpha^{(m)}\right)_{c}^{(m)}\left(-\beta_{01}^{(m)}-\beta_{\infty}^{(m)}, \omega\right)\right]} \\
\mu_{s}^{(m)}= & -\mu_{0}^{(m)} \frac{3}{2\left(1+\nu_{0}^{(m)}\right)}\left(d^{(m)}+\frac{3 \beta_{0}^{(m)}}{2\left(1+\nu_{0}^{(m)}\right)}\right)^{-1} \\
& R_{\alpha^{(m)}(m)}^{(m)}\left(-\beta_{01}^{(m)}-\beta_{\infty}^{(m)}, \omega\right),
\end{aligned}
$$

where

$$
\begin{aligned}
& R_{\alpha^{(m)} c}^{(m)}=\frac{\left(\xi^{(m)}\right)^{2}+\xi^{(m)} \sin \left(\pi \alpha^{(m)} / 2\right)}{\left(\xi^{(m)}\right)^{2}+2 \xi^{(m)} \sin \left(\pi \alpha^{(m)} / 2\right)+1} \\
& R_{\alpha^{(m)} s}^{(m)}=\frac{\xi^{(m)} \cos \left(\pi \alpha^{(m)} / 2\right)}{\left(\xi^{(m)}\right)^{2}+2 \xi^{(m)} \sin \left(\pi \alpha^{(m)} / 2\right)+1} \\
& \xi^{(m)}=\left(Q^{(m)} \Omega\right)^{\alpha^{(m)}-1}, \quad \Omega=k_{1} h \frac{c}{c_{2}^{(1)}} .
\end{aligned}
$$

At the same time, as in the papers by Akbarov (2014) and Akbarov and Kepceler (2015) we introduce the following dimensionless rheological parameters:

$$
d^{(m)}=\frac{\beta_{\infty}^{(m)}}{\beta_{0}^{(m)}}, \quad Q^{(m)}=\frac{\beta_{\infty}^{(m)}}{R\left(\beta_{0}^{(m)}+\beta_{\infty}^{(m)}\right)^{1 /\left(1-\alpha^{(m)}\right)}} .
$$

It is difficult to give concrete physico-mechanical meaning to the rheological parameters $\beta_{0}^{(m)}$ and $\beta_{\infty}^{(m)}$; however, the dimensionless parameters $d^{(m)}$ and $Q^{(m)}$ have real rheological meaning. The dimensionless rheological parameter $d^{(m)}$ 
characterises the long-term value of the elastic constants, the parameter $Q^{(m)}$ characterises the creep time and the rheological parameter $\alpha^{(m)}$ characterises the form of the creep (or relaxation) function of the viscoelastic material at the beginning region of deformation. Note that the case where $\alpha^{(m)}=0$ corresponds to the 'standard viscoelastic body' model. Consequently, the effect of the viscoelasticity of the material on the attenuation curves will be estimated through these three dimensionless

rheological parameters.

For the long-term values of the mechanical constants $\lambda_{c}^{(m)}, \lambda_{s}^{(m)}, \mu_{c}^{(m)}$ and $\mu_{s}^{(m)}$, which enter into equation (13), the following expressions can be obtained:

$$
\begin{aligned}
\lambda_{\infty}^{(m)}= & \lim _{t \rightarrow \infty}\left(\lambda^{(m) *} \cdot 1\right) \\
= & \lambda_{0}^{(m)}\left[1+\frac{\left(1-2 \nu_{0}^{(m)}\right)}{2 \nu_{0}^{(m)}\left(1+\nu_{0}^{(m)}\right)}\right. \\
& \left.\frac{1}{\left(3 /\left(2\left(1+\nu_{0}^{(m)}\right)\right)+d^{(m)}\right.}\right], \\
\mu_{\infty}^{(m)}= & \lim _{t \rightarrow \infty}\left(\mu^{(m) *} \cdot 1\right) \\
= & \mu_{0}^{(m)}\left[1-\frac{3}{2\left(1+\nu_{0}^{(m)}\right)}\right. \\
& \left.\frac{1}{\left(3 /\left(2\left(1+\nu_{0}^{(m)}\right)\right)+d^{(m)}\right.}\right] .
\end{aligned}
$$

This completes the selection of the viscoelastic operators and dimensionless viscoelastic operators.

Note that one of the advantages of the fractionalexponential operator is its ability to describe the initial parts of the experimentally constructed creep and relaxation graphs and their asymptotic values with very high accuracy. It should be noted that there are also other types of fractional order operators (see, for instance, Sawicki and Padovan 1999; Adolfson et al. 2005 and others listed therein) which differ from Rabotnov's fractional-exponential operator.

The experimental validations of the fractionalexponential operators (17) and determination of the rheological parameters $\alpha, \beta_{\infty}$ and $\beta_{0}$ entering these operators can be found in many investigations carried out by Rabotnov (1980), Kaminskii and Selivanov (2005) and Golub et al. (2008). For example, it is experimentally established (see Golub et al. 2008) that the rheological parameters of the polymer concrete are $\alpha=0.723778, \beta_{0}+$ $\beta_{\infty}=0.18875 h^{\alpha-1}$ and $\beta_{0}=0.02598 h^{\alpha-1}$ and the rheological parameters of polymethyl methacrylate are: $\alpha=0.53, \quad \beta_{0}+\beta_{\infty}=49$ day $^{1-\alpha}$ and $\beta_{0}=0.98$ day $^{1-\alpha}$, which are also determined experimentally and discussed in the paper by Kaminskii and Selivanov (2005). Therefore, these and other examples allow us to conclude that, through operators (17 and 18), the constituent relationships can be described for a very wide range of linear viscoelastic materials with the corresponding rheological values $\alpha, \beta_{\infty}$ and $\beta_{0}$.

\subsection{Algorithm for the determination of the attenuation curves}

First, note that as we consider the time harmonic wave propagation in a viscoelastic material, we must therefore assume that the wavenumber $k$ is a complex number and can be presented as follows:

$$
k=k_{1}+\mathrm{i} k_{2}=k_{1}(1+\mathrm{i} \beta), \quad \beta=\frac{k_{2}}{k_{1}} .
$$

Here, the imaginary part $k_{2}$ of the wavenumber $k$ (or parameter $\beta$ called the attenuation coefficient) defines the amplitude attenuation of the considered wave. Note that we determine the phase velocity of the studied waves through the expression $c=\omega / k_{1}$.

Considering relations (22-24) and according to the known physico-mechanical considerations, one can predict that in the case where $\left(Q^{(m)} \Omega\right) \ll$ 1 , the behaviour of the viscoelastic system must be very close to the corresponding purely elastic case with long-term values of the elastic constants at $t=\infty$. In the same way, in the case where $\left(Q^{(m)} \Omega\right) \gg 1$, its behaviour must be very close to the corresponding purely elastic system with instantaneous values of the elastic constants at $t=0$. Accordingly, it can be assumed that increasing the values of the $Q^{(m)}$ and $d^{(m)}$ parameters corresponds to reducing the viscous part of the viscoelastic deformations in the constituents. As a result, by decreasing the values of the rheological parameters $Q^{(m)}$ and $d^{(m)}$, we increase the effect of the viscosity of the material on the dispersion curves.

Considering the solution of dispersion equation (16) and as the determinant values are complex, the dispersion equation can be reduced to the following form:

$$
\left|\operatorname{det}\left\|\alpha_{i j}\right\|\right|=0
$$


where $\left|\operatorname{det}\left\|\alpha_{i j}\right\|\right|$ is the modulus of the complex number det $\left\|\alpha_{i j}\right\|$. Consequently, to construct the attenuation or dispersion curves, it is necessary to solve equation (25) numerically for the selected parameters of the problem.

The dispersion equation contains three unknowns: $c, k_{1} h$ and $\beta$, therefore, the values of two unknowns must be given in advance to determine the value of the remaining third from the dispersion equation. If the selected two unknowns are $k_{1} h$ and $\beta$, then we can determine the wave propagation velocity $c$ as a result of the solution of the dispersion equation. Note that this approach has already been used in the papers by Akbarov and Kepceler (2015), Akbarov et al. (2016a,b) and Akbarov and Negin (2017b) in which the corresponding expressions given in the references in Ewing et al. (1957) and Kolsky (1963) are used to determine the attenuation coefficient of waves. However, if the selected two unknowns are $c$ and $k_{1} h$, then we can determine the attenuation coefficient $\beta$ from the dispersion equation, which is the procedure used in the present paper to determine the attenuation curves. Note that the latter approach was also made in the papers by Barshinger and Rose (2004) and Kocal and Akbarov (2017).

With respect to the sign of the dispersion equation, as in the viscoelastic case $\left|\operatorname{det}\left\|\alpha_{i j}\right\|\right| \geq 0$, we cannot use the algorithm based on the sign change of the determinant to find the roots of the dispersion equation. Therefore, in the viscoelastic case, we use the algorithm based on the direct calculation of the values of the modulus of the dispersion determinant det $\left\|\alpha_{i j}\right\|$ and the sought roots are determined from the criterion $\left|\operatorname{det}\left\|\alpha_{i j}\right\|\right| \leq$ $10^{-9}$.

It should be noted that there is no general method for finding the complex roots of the transcendental secular equations (see, for instance, Sharma 2011). The functional iteration method described by Sharma (2011) can be applied for the determination of the complex roots of an analytical function. This method is used, in particular, to solve the complex roots of the corresponding secular equations in Sharma (2005), Sharma and Othman (2007), Kumar and Parter (2009), Sharma and Kumar (2009), Sharma et al. (2009) and others listed therein. Even though the functional iteration method makes it possible to simultaneously determine the real and imaginary parts of the complex roots of the secular equations, the algorithm detailed above and which is used in the present paper does not require a reduction of the secular equation to the corresponding algebraic equation and the successful selection of the initial iterations.

It should be noted that the results obtained by Barshinger and Rose (2004), using the same method as in this paper, are confirmed by the relevant experimental data, which is crucial to demonstrate the relevance of this method.

\subsection{Numerical results and their discussions}

We turn to the analysis of the numerical results for a detailed illustration of the foregoing algorithm obtained under the assumptions that $\nu_{0}^{(1)}=\nu_{0}^{(2)}=$ $0.3, \rho^{(1)}=2.8 \mathrm{~g} / \mathrm{cm}^{3}, \rho^{(2)}=3.2 \mathrm{~g} / \mathrm{cm}^{3}, c_{2}^{(1)}=$ $3 \mathrm{~km} / \mathrm{s}$ and $c_{2}^{(2)}=5 \mathrm{~km} / \mathrm{s}$, where $\nu_{0}^{(1)}, \rho^{(1)}$ and $c_{2}^{(1)}$ are the Poisson ratio, mass density and shear wave velocity of the elastic layer and $\nu_{0}^{(2)}, \rho^{(2)}$ and $c_{2}^{(2)}$ are the Poisson ratio, mass density and the shear wave velocity of the viscoelastic half-space, respectively. Note that we consider the results obtained in the case where the material of the covering layer is purely elastic, but the material of the half-space is viscoelastic. Thus, in the case where the material of the covering layer is purely elastic, the operators $\lambda^{(1)^{*}}$ and $\mu^{(1)^{*}}$ in the preceding expressions and equations are replaced with the corresponding Lame constants $\lambda_{0}^{(1)}$ and $\mu_{0}^{(1)}$, respectively. Consequently, the influence of the viscoelasticity of the material on the dispersion curves is estimated through three dimensionless rheological parameters of the viscoelastic half-space material, i.e., the dimensionless rheological parameter $d\left(=d^{(2)}\right)$ which characterises the long-term value, the rheological parameter $Q\left(=Q^{(2)}\right)$ which characterises the creep time of the viscoelastic half-space material and finally the rheological parameter $\alpha\left(=\alpha^{(2)}\right)$ which characterises the form of the creep (or relaxation) function of this material. In this paper, we assume that $\alpha=0.5$ throughout the numerical investigations.

Note that the numerical results detailed in the paper by Akbarov and Negin (2017b), as well as in many others that are not given here, show that the dispersion curves obtained for the corresponding purely elastic cases with instantaneous and long-term values of the elastic constants can be considered as the lower and upper limit cases of the dispersion curves obtained for the considered viscoelastic case. Namely, this statement allows us to select admissible dispersion curves and the corresponding wave propagation velocity $c$ for the viscoelastic case. Then using these curves, we can 
find the corresponding attenuation coefficient $\beta$ from the solution of dispersion equation (25) for each fixed value of the dimensionless wavenumber.

Thus, we first construct dispersion curves for the purely elastic case with instantaneous and longterm values of the elastic constants of the covering layer and of the half-space materials. These dispersion curves are illustrated by dashed lines in figure 2 . In this way, according to the previous discussions, after the construction of the dispersion

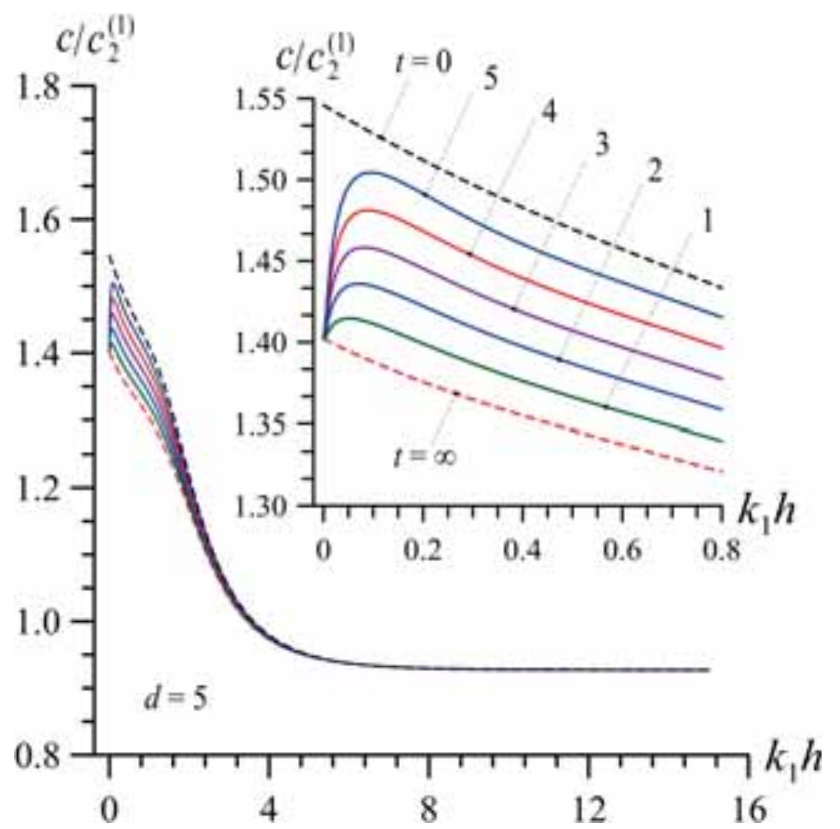

Figure 2. Selected dispersion curves for the case where $d=5$.

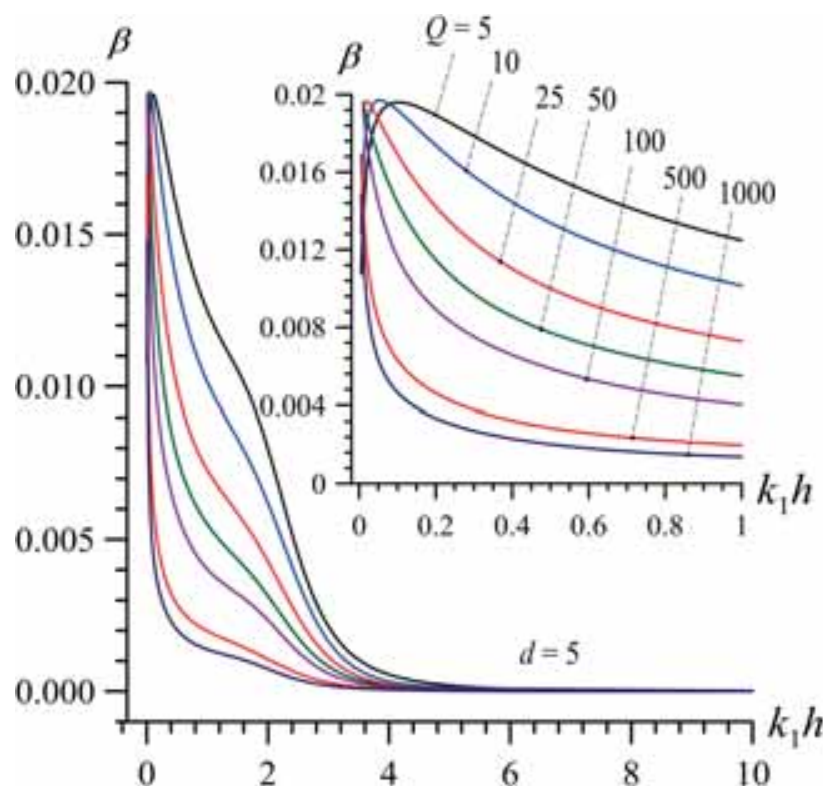

Figure 3. Attenuation of curve number 1 for different values of $Q$ in figure 2 . curves related to the purely elastic cases, we can now select the admissible dispersion curves related to the different viscoelastic cases. For example, we can take the dispersion curves shown in figure 2 with solid lines numbered $1-5$ from the dispersion curve constructed at $t=\infty$ and at $t=0$.

Following the above preparation, we now choose the values for the dimensionless wavenumber $k_{1} h$ and the wave propagation velocity $c / c_{2}^{(1)}$ according to the admissible dispersion curves indicated in

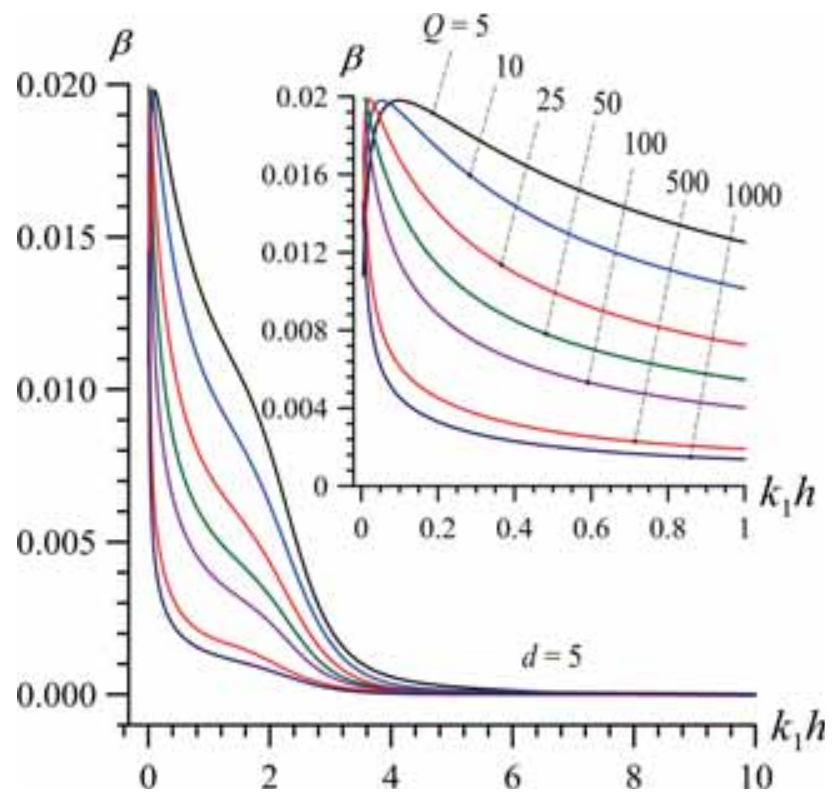

Figure 4. Attenuation of curve number 2 for different values of $Q$ in figure 2 .

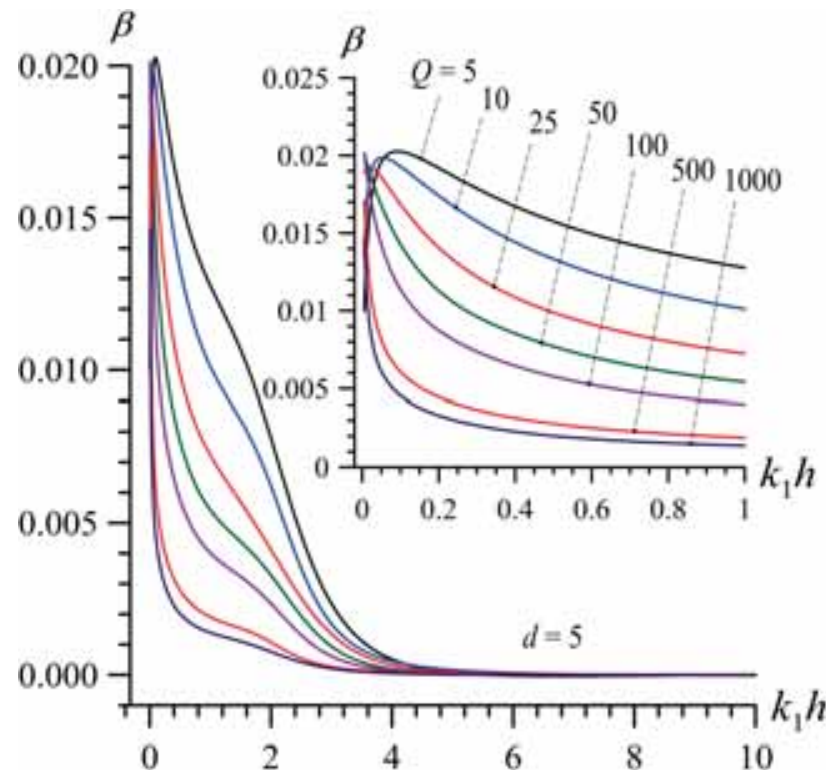

Figure 5. Attenuation of curve number 3 for different values of $Q$ in figure 2 . 
figure 2. For example, if we take the dispersion curve indicated by number 1 in figure 2 as an admissible dispersion curve, then for the given wavenumber $k_{1} h$, the values of the wave propagation velocities $c / c_{2}^{(1)}$ are determined from this curve. After this determination, we finally calculate the attenuation coefficient $\beta$ from dispersion equation (25) given certain values for the

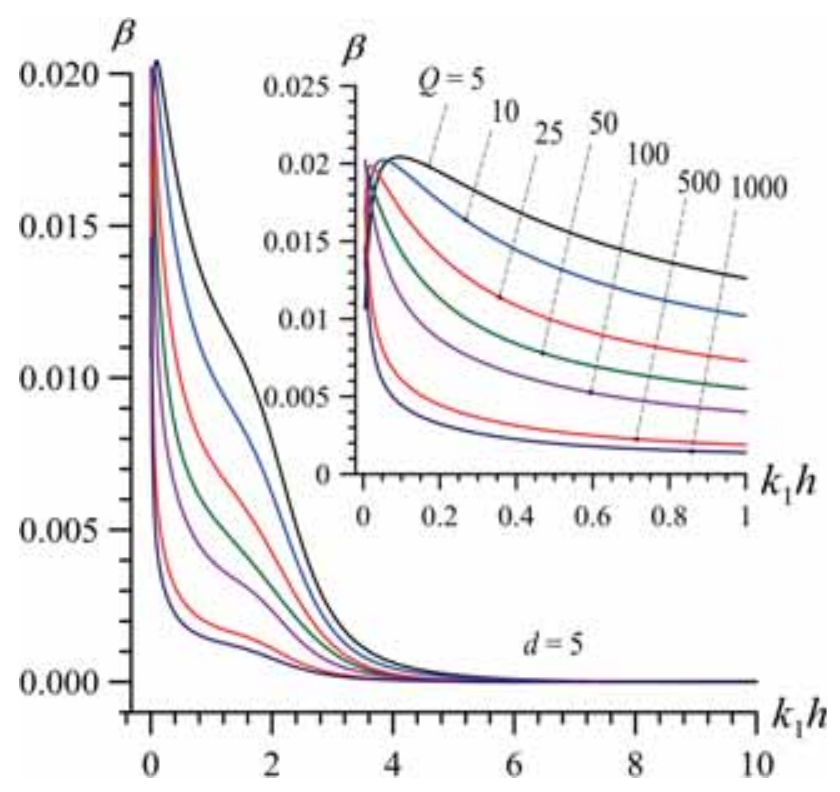

Figure 6. Attenuation of curve number 4 for different values of $Q$ in figure 2 .

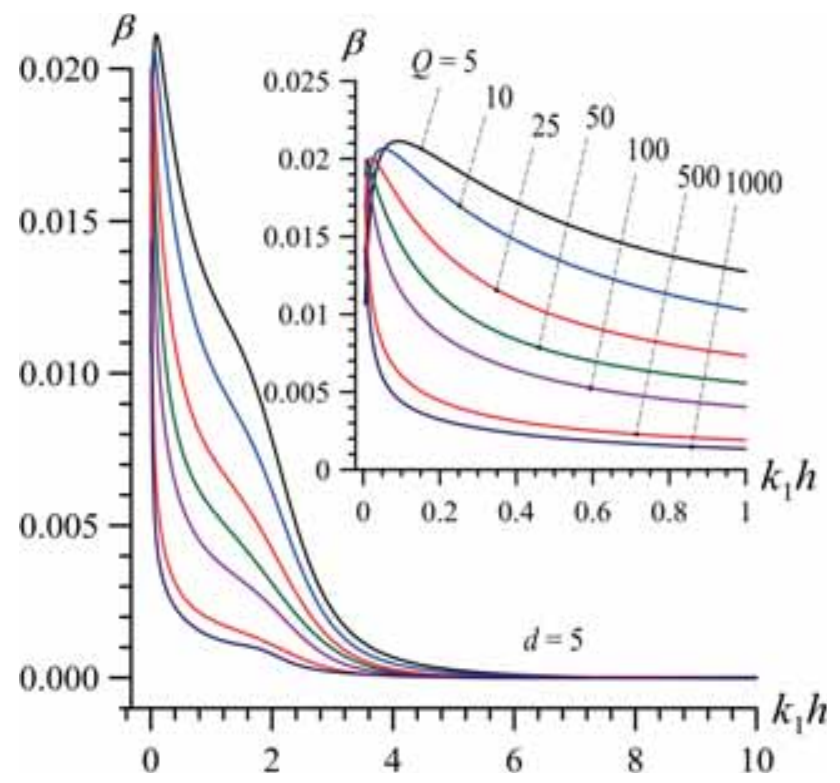

Figure 7. Attenuation of curve number 5 for different values of $Q$ in figure 2 . rheological parameters $Q, d$ and $\alpha$, and so construct the attenuation curves which will be discussed below.

Thus, by employing the above solution procedure we find the attenuation curves shown in figures 3-7 which are constructed under different values of the parameter $Q$ for the dispersion curves indicated by numbers $1,2,3,4$ and 5 in figure 2 , respectively.

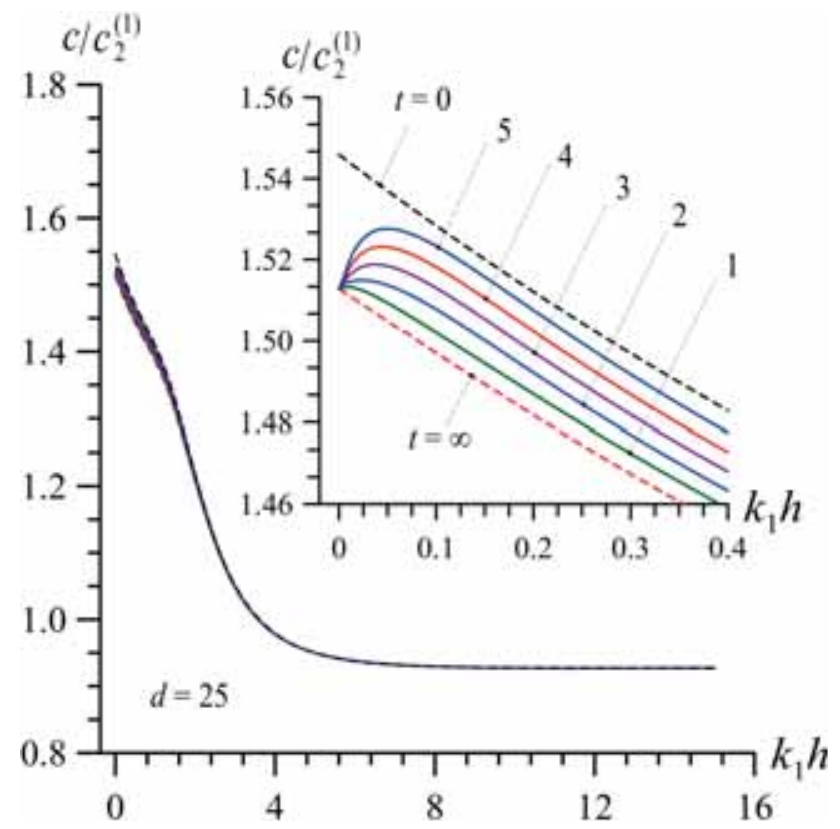

Figure 8. Selected dispersion curves for the case where $d=$ 25 .

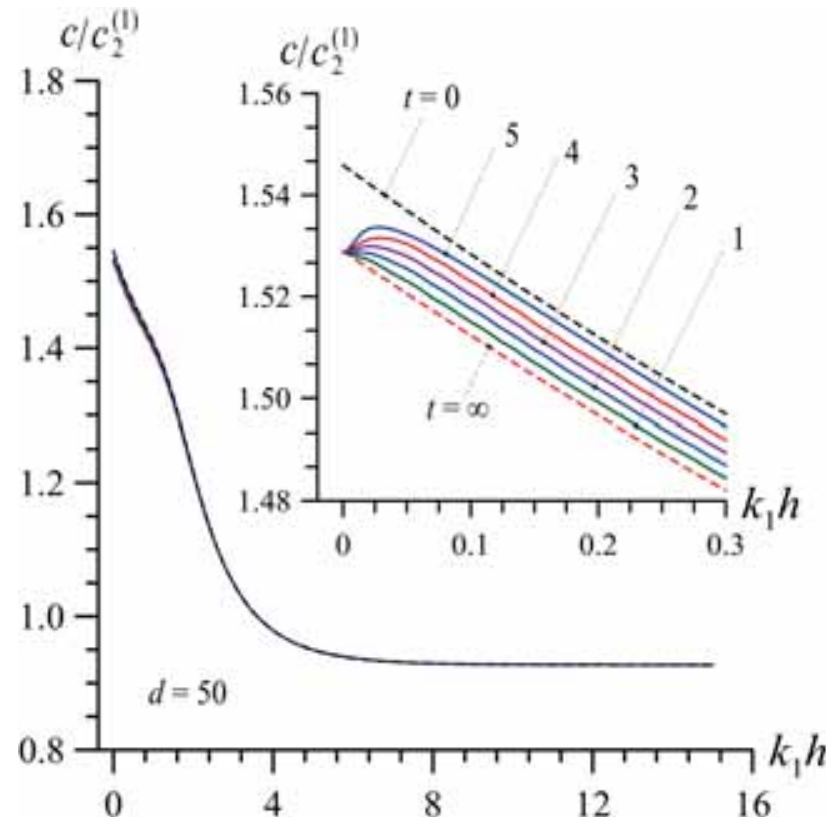

Figure 9. Selected dispersion curves for the case where $d=$ 50 . 


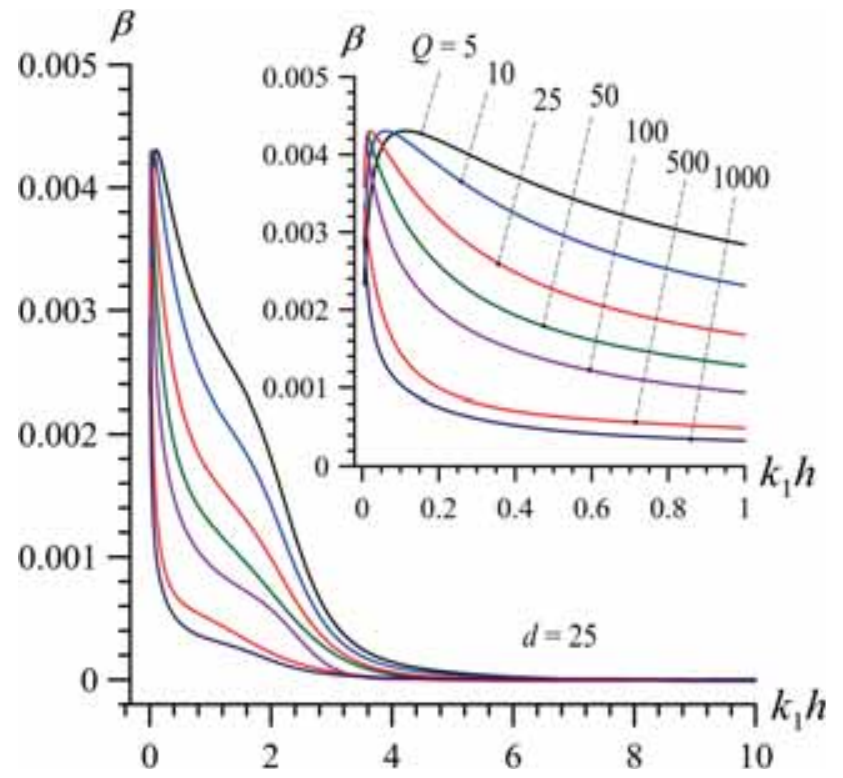

Figure 10. Attenuation of curve number 1 for different values of $Q$ in figure 8 .

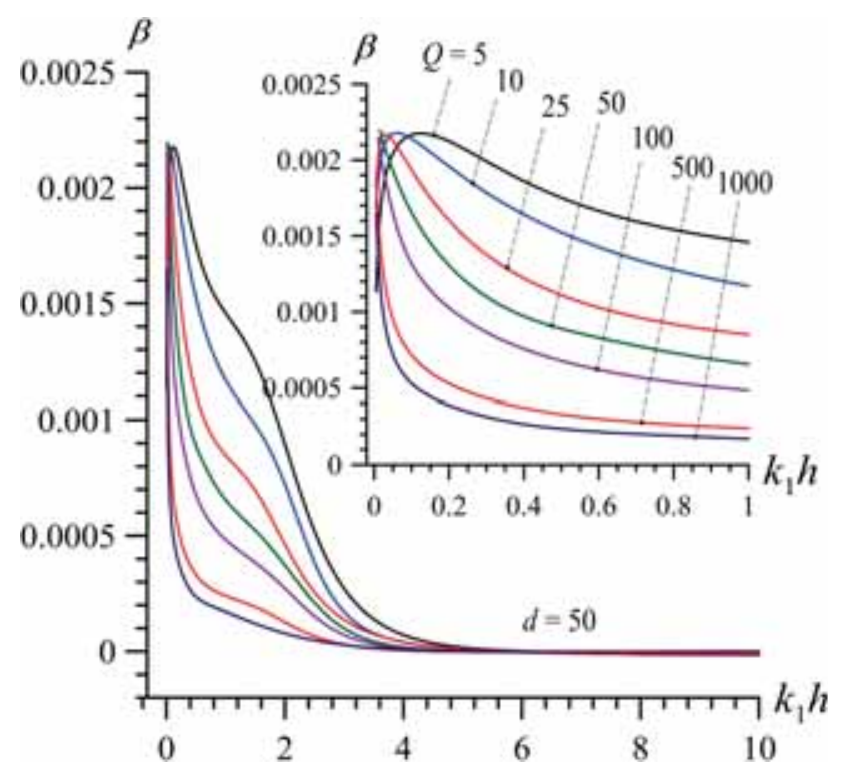

Figure 11. Attenuation of curve number 1 for different values of $Q$ in figure 10 .

Note that the attenuation curves shown in figures 3-7 are obtained in the case where $d=5$. To illustrate the influence of the rheological parameter $d$ on the attenuation curves, similar results are also obtained for far greater values of parameter $d$, namely, $d=25, d=50$ and the dispersion curves are illustrated in figures 8 and 9 , respectively. The attenuation curves related to the dispersion curve indicated by number 1 in figures 8 and 9 are shown in figures 10 and 11, to give some examples. However, not all attenuation curves associated with these cases are given here to reduce the volume of the paper.

The concrete conclusions following from the foregoing numerical results are given in the next section.

\section{Conclusions}

We have proposed an approach to determine the attenuation coefficient of seismic Rayleigh waves propagating in an elastic crustal layer over the viscoelastic mantle (half-space). The approach is based on the selection of the admissible dispersion curves of the Rayleigh waves that can propagate in the considered elastic--viscoelastic covering halfspace. The investigations are carried out within the framework of the exact equations of motion of the theory of linear viscoelasticity. The constitutive relations of the viscoelastic material of the half-space are described by Rabotnov (1980) using fractional-exponential operators. Three dimensionless rheological parameters are introduced and, through these parameters, the influence of the viscosity of the covered half-space material on the attenuation curves is studied. The numerical results related to those curves are presented and from the results the following concrete conclusions are obtained:

- considerable attenuation of the amplitudes of the quantities related to the stress-strain state is observed for the low wavenumber cases;

- increasing the values of the $d$ and $Q$ rheological parameters leads to a decrease in the values of the attenuation coefficient;

- the decreasing rate of the attenuation coefficient (with respect to the wavenumber) increases with the rheological parameter $Q$;

- after a certain value of the dimensionless wavenumber $\left(\right.$ say $\left.\left(k_{1} h\right)^{*}\right)$, the values of the attenuation coefficient decrease monotonically with $k_{1} h$;

- the value of $\left(k_{1} h\right)^{*}$ depends on the rheological parameters $d$ and $Q$ and

- the effect of the 'distance' of the dispersion curves from the elastic limit curves on the attenuation coefficient is insignificant.

In addition to the above conclusions, these results can also be used in the theoretical sense. For example, using these results, it can be predicted how an increase or decrease of the rheological parameters such as the creep time or the long-term values of the mechanical properties of certain polymerviscoelastic materials can affect (qualitatively) the 
wave attenuation of the elements made from these materials. Moreover, these results make it possible to predict how changing the geometric sizes of the system under consideration may affect the influence of the material viscosity on wave attenuation, which helps in understanding the nature of the propagation and attenuation processes of the waves in layered-viscoelastic half-spaces.

\section{Acknowledgements}

This research received no specific grant from any funding agency in the public, commercial, or notfor-profit sectors.

\section{References}

Addy S K and Chakraborty N R 2005 Rayleigh waves in a viscoelastic half-space under initial hydrostatic stress in presence of the temperature field; Int. J. Math. Sci. 2005(24) 3883-3894.

Adolfson K, Enelund M and Olsson P 2005 On the fractional order model of viscoelasticity; Mech. Time-Depend Mater. 9 15-34.

Akbarov S D 2014 Axisymmetric time-harmonic Lamb's problem for a system comprising a viscoelastic layer covering a viscoelastic half-space; Mech. Time-Depend Mater. 18(1) 153-178.

Akbarov S D 2015 Dynamics of pre-strained bi-material elastic systems: Linearized three-dimensional approach; Springer International, Switzerland, https://doi.org/10. 1007/978-3-319-14460-3.

Akbarov S D and Kepceler T 2015 On the torsional wave dispersion in a hollow sandwich circular cylinder made from viscoelastic materials; Appl. Math. Model. 39(13) 3569-3587.

Akbarov S D and Negin M 2017a Near-surface waves in a system consisting of a covering layer and a half-space with imperfect interface under two-axial initial stresses; J. Vib. Control 23(1) 55-68.

Akbarov S D and Negin M 2017b Generalized Rayleigh wave dispersion in a covered half-space made of viscoelastic materials; CMC-Comput. Mater. Con. 53(4) 307-341.

Akbarov S D, Kocal T and Kepceler T 2016a On the dispersion of the axisymmetric longitudinal wave propagating in a bi-layered hollow cylinder made of viscoelastic materials; Int. J. Solids Struct. 100 195-210.

Akbarov S D, Kocal T and Kepceler T 2016b Dispersion of axisymmetric longitudinal waves in a bi-material compound solid cylinder made of viscoelastic materials; CMC-Comput. Mater. Con. 51(2) 105-143.

Aki K and Richards P G 2002 Quantitative seismology (2nd edn); University Science Books.

Barshinger J N and Rose J L 2004 Guided wave propagation in an elastic hollow cylinder coated with a viscoelastic material; IEEE Trans. Ultrason. Ferroelectr. 51(11) $1547-1556$.
Bosiakov S M 2014 On the application of a viscoelastic model with Rabotnov's fractional exponential function for assessment of the stress-strain state of the periodontal ligament; Int. J. Mech. 8 353-358.

Carcione J M 1992 Rayleigh waves in isotropic viscoelastic media; Geophys. J. Int. 108(2) 453-464.

Carcione J M 1995 Constitutive model and wave equations for linear, viscoelastic, anisotropic media; Geophysics 60(2) 537-548.

Carcione J M 2007 Wave fields in real media: Wave propagation in anisotropic, anelastic, porous and electromagnetic media; Vol. 38, Elsevier, Amsterdam.

Carcione J M, Poletto F and Gei D 2004 3-D wave simulation in anelastic media using the Kelvin-Voigt constitutive equation; J. Comput. Phys. 196(1) 282-297.

Castaings M and Hosten B 2003 Guided waves propagating in sandwich structures made of anisotropic, viscoelastic, composite materials; J. Acoust. Soc. Am. 113(5) 2622-2634.

Chen Z J, He Y and Gao J 2015 On the comparison of properties of Rayleigh waves in elastic and viscoelastic media; Int. J. Numer. Anal. Mod. 12(2) 254-267.

Chiriţă S, Ciarletta M and Tibullo V 2014 Rayleigh surface waves on a Kelvin-Voigt viscoelastic half-space; $J$. Elast. 115(1) 61-76.

Eldred L B, Baker W P and Palazotto A N 1995 KelvinVoigt versus fractional derivative model as constitutive relations for viscoelastic materials; AIAA J. 33(3) $547-$ 550.

Ely G P, Day S M and Minster J B 2008 A support-operator method for viscoelastic wave modelling in 3-D heterogeneous media; Geophys. J. Int. 172(1) 331-344.

Ewing W M, Jardetzky W S, Press F and Beiser A 1957 Elastic waves in layered media; Phys. Today 1027.

Fan J 2004 Surface seismic Rayleigh wave with nonlinear damping; Appl. Math. Model. 28(2) 163-171.

Garg N 2007 Effect of initial stress on harmonic plane homogeneous waves in viscoelastic anisotropic media; J. Sound. Vib. 303(3) 515-525.

Golub V P, Fernati P V and Lyashenko Y G 2008 Determining the parameters of the fractional exponential heredity kernels of linear viscoelastic materials; Int. Appl. Mech. 44(9) 963-974.

Ivanov T P and Savova R 2014 Motion of the particles due to viscoelastic surface waves of an assigned frequency; Math. Mech. Solids 19(6) 725-731.

Jiangong Y 2011 Viscoelastic shear horizontal wave in graded and layered plates; Int. J. Solids Struct. 48(16) 2361-2372.

Jousset P, Neuberg J and Jolly A 2004 Modelling lowfrequency volcanic earthquakes in a viscoelastic medium with topography; Geophys. J. Int. 159(2) 776-802.

Kaminskii A A and Selivanov M F 2005 An approach to the determination of the deformation characteristics of viscoelastic materials; Int. Appl. Mech. 41(8) 867-875.

Kielczyriski P and Cheeke J D N 1997 Love waves propagation in viscoelastic media [and NDT application]; In: Proceedings of the IEEE ultrasonics symposium, 1997, Vol. 1, pp. 437-440.

Kocal T and Akbarov S D 2017 On the attenuation of the axisymmetric longitudinal waves propagating in the 
bi-layered hollow cylinder made of viscoelastic materials; Struct. Eng. Mech. 61(2) 145-165.

Kolsky H 1963 Stress waves in solids; Vol. 1098, Courier Corporation, North Chelmsford.

Kumar R and Parter G 2009 Analysis of free vibrations for Rayleigh-Lamb waves in a microstretch thermoelastic plate with two relaxation times; J. Eng. Phys. Thermophys. 82 35-46.

Lai C G and Rix G J 2002 Solution of the Rayleigh eigen problem in viscoelastic media; Bull. Seismol. Soc. Am. 92(6) 2297-2309.

Manconi E and Sorokin S 2013 On the effect of damping on dispersion curves in plates; Int. J. Solids. Struct. 50(11) 1966-1973.

Meral F C, Royston T J and Magin R L 2009 Surface response of a fractional order viscoelastic halfspace to surface and subsurface sources; J. Acoust. Soc. Am. 126(6) 3278-3285.

Meral F C, Royston T J and Magin R L 2011 Rayleigh-lamb wave propagation on a fractional order viscoelastic plate; J. Acoust. Soc. Am. 129(2) 1036-1045.

Negin M 2015 Generalized Rayleigh wave propagation in a covered half-space with liquid upper layer; Struct. Eng. Mech. 56(3) 491-506.

Negin M, Akbarov S D and Erguven M E 2014 Generalized Rayleigh wave dispersion analysis in a pre-stressed elastic stratified half-space with imperfectly bonded interfaces; CMC-Comput. Mater. Con. 42(1) 25-61.

Pasternak M 2008 New approach to Rayleigh wave propagation in the elastic halfspace-viscoelastic layer interface; Acta Phys. Pol. A $\mathbf{1 1 4}(\mathbf{6 A})$.

Quintanilla F H, Fan Z, Lowe M J S and Craster R V 2015 Guided waves' dispersion curves in anisotropic viscoelastic single-and multi-layered media; Int. Proc. $R$. Soc. A 471(2183) 20150268.

Rabotnov Y N 1980 Elements of hereditary solid mechanics; Mir, Moscow.

Romeo M 2001 Rayleigh waves on a viscoelastic solid halfspace; J. Acoust. Soc. Am. 110(1) 59-67.

Rossikhin Y A 2010 Reflections on two parallel ways in the progress of fractional calculus in mechanics of solids; Appl. Mech. Rev. 63(1) 010701-1-12.
Rossikhin Y A and Shitikova M V 2014 The simplest models of viscoelasticity involving fractional derivatives and their connectedness with the Rabotnov fractional order operators; Int. J. Mech. 8 326-331.

Sawicki J T and Padovan J 1999 Frequency driven phasic shifting and elastic-hysteretic portioning properties of fractional mechanical system representation schemes; $J$. Franklin Inst. 336 423-433.

Sharma J N 2005 Some considerations on the Rayleigh Lamb waves in viscoelastic plates; J. Vib. Control 11 1311-1335.

Sharma M D 2011 Phase velocity and attenuation of plane waves in dissipative elastic media: Solving complex transcendental equation using functional iteration method; Int. J. Eng. Sci. Technol. 3(2) 130-136.

Sharma J N and Kumar S 2009 Lamb waves in micropolar thermoelastic solid plates immersed in liquid with varying temperature; Mechanics 44 305-319.

Sharma J N and Othman M I A 2007 Effect of rotation on generalized thermo-viscoelastic Rayleigh-Lamb waves; Int. J. Solids Struct. 44 4243-4255.

Sharma J N, Sharma R and Sharma P K 2009 Rayleigh waves in a thermo-viscoelastic solid loaded with viscous fluid of varying temperature; Int. J. Theor. Appl. Sci. 1(2) 60-70.

Simonetti F and Cawley P 2003 A guided wave technique for the characterization of highly attenuative viscoelastic materials; J. Acoust. Soc. Am. 114(1) $158-165$.

Vishwakarma S K and Gupta S 2012 Torsional surface wave in a homogeneous crustal layer over a viscoelastic mantle; Int. J. Appl. Math. Mech. 8(16) 38-50.

Yuan S, Song X, Cai W and Hu Y 2018 Analysis of attenuation and dispersion of Rayleigh waves in viscoelastic media by finite-difference modeling; J. Appl. Geophys. 148 $115-126$.

Zhang K, Luo Y, Xia J and Chen C 2011 Pseudospectral modeling and dispersion analysis of Rayleigh waves in viscoelastic media; Soil Dyn. Earthq. Eng. 31(10) 1332-1337.

Zhou Y 2009 Surface-wave sensitivity to 3-D anelasticity; Geophys. J. Int. 178(3) 1403-1410 